\title{
ACCURATE ITERATIVE ANALYSIS OF THE K-V EQUATIONS
}

\section{O. A. Anderson, Lawrence Berkeley National Laboratory, 1 Cyclotron Road, Berkeley, CA 94720 USA}

Those working with alternating-gradient (A-G) systems look for simple, accurate ways to analyze A-G performance for matched beams. The useful $\mathrm{K}-\mathrm{V}$ equations [1] are easily solved in the smooth approximation [2], [3], [4]. This approximate solution becomes quite inaccurate for applications with large focusing fields and phase advances. Results of efforts to improve the accuracy [5], [6] have tended to be indirect or complex. Our generalizations presented previously [7] gave better accuracy in a simple explicit format. However, the method used to derive our results (expansion in powers of a small parameter) was complex and hard to follow; also, reference [7] only gave low-order correction formulas.

The present paper uses a straightforward iteration method and obtains equations of higher order than shown in our previous paper.

The K-V equations for the envelopes $a(z)$ and $b(z)$ are

$$
\begin{aligned}
& a(z)^{\prime \prime}=-K(z) a+\frac{E^{2}}{a^{3}}+\frac{2 Q}{a+b} \\
& b(z)^{\prime \prime}=+K(z) b+\frac{E^{2}}{b^{3}}+\frac{2 Q}{a+b}
\end{aligned}
$$

with input parameters: normalized beam current $Q$; emittance $E$; and $A-G$ focus function $K(z)$. The $z$ origin is located at the midpoint of a quadrupole and $\mathrm{K}(\mathrm{z})$ is assumed here to be symmetric about $z=0$, periodic over a cell length $2 L$, and antisymmetric about $L / 2$. Thus

$$
\mathrm{K}(\mathrm{z}-2 \mathrm{~L})=\mathrm{K}(\mathrm{z}), \mathrm{K}(-\mathrm{z})=\mathrm{K}(\mathrm{z}), \mathrm{K}(\mathrm{z}-\mathrm{L})=-\mathrm{K}(\mathrm{z}) .
$$

We solve for the $x$ and $y$ beam envelopes $a(z)$ and $b(z)$ assumed to be matched to the lattice, i.e., periodic over $2 \mathrm{~L}$. To aid the solution of Eqs. (1) and (2), we define in Eqs. (4)-(19) the operators on even periodic functions $\langle\ldots\rangle,\{\ldots\}, \int$ and $\left.\int\right]$; the even periodic functions $h(z), g(z), \delta(z)$ and $\rho(z) ;$ and the constants $\mathrm{k}, \alpha, \beta, \mathrm{q}, \mathrm{A}, \mathrm{K}_{\mathrm{eff}}, \Phi$, and $\mathrm{p}_{\mathrm{m}}$. In Eq. (19), $\mathrm{h}_{1}$ is the first Fourier coefficient of $\mathrm{h}(\mathrm{z})$.

The operator $(\ldots)$ performs an average over a cell length $2 \mathrm{~L}$ while the operator $\{\ldots\}$ removes the average part of a periodic

Table 1: Definitions to be used in this paper

$$
\begin{aligned}
& \langle f\rangle \equiv(1 / 2 L) \int_{0}^{2 L} f(z) d z \\
& \{f\} \equiv f-\langle f\rangle \\
& \text { For even } \psi(z) \ni\langle\psi\rangle=0: \\
& \int \Psi \equiv \int_{0}^{z} \psi\left(z^{\prime}\right) d z^{\prime} \quad \text { and } \\
& \iint \psi \equiv\left\{\int_{0}^{z} d z^{\prime} \int_{0}^{z^{\prime}} \Psi\left(z^{\prime \prime}\right) d z^{\prime \prime}\right\} \\
& k \equiv K^{\max }, \\
& h(z) \equiv K(z) / k \\
& g \equiv \iint h,
\end{aligned}
$$$$
\text { (4) } \delta(\mathrm{z}) \equiv \iint\{\mathrm{hg}\} \text {, }
$$$$
A \equiv\langle\mathrm{a}(\mathrm{z})\rangle \text {, }
$$

function: e.g., $2\left\{\cos ^{2} x\right\}=\{1+\cos 2 x\}=\cos 2 x$. The operator $\iint$ operates on periodic functions that have no average. It gives the repeated indefinite integral and removes the average part, if any, of the result.

\section{DECOUPLING AND DECOMPOSING}

With the quadrupole symmetries of Eq. (3), our matched beam assumption implies $b(z)=a(z+L)$, so that Eqs. (1) and (2) are decoupled. We have $\langle a\rangle=\langle b\rangle \equiv A$, and

$$
a=A(1+p), \quad b=A\left(1+\rho_{b}\right) .
$$

The $Q$ terms in Eqs. (1) and (2) can be expanded as

$$
\frac{2 \mathrm{Q}}{\mathrm{a}+\mathrm{b}}=\frac{\mathrm{Q}}{\mathrm{A}}\left(1-\left(\mathrm{p}+\mathrm{p}_{\mathrm{b}}\right) / 2+\ldots\right)=\frac{\mathrm{Q}}{\mathrm{A}}\left(1-\mathrm{k}^{2} \delta(\mathrm{z})+\ldots\right) \text {, }
$$

since [8]

$$
\left(p+p_{b}\right) / 2=k^{2} \delta(z)+\ldots .
$$

with $\delta(\mathrm{z})$ [Eq. (11)] derived from the lattice waveform $\mathrm{h}(\mathrm{z})$.

This decouples Eqs. (1) and (2). After the decoupled version of Eq. (1) is solved for $a(z)$, then $b(z)$ is found by symmetry. Equation (2) is no longer needed.

Substituting $a=A(1+\rho)$ in the first three terms of Eq. (1), expanding $1 / \mathrm{a}^{3}$, dividing by $A$, and using (21) and (15), the first $\mathrm{K}-\mathrm{V}$ equation is equivalent to

$$
\begin{array}{r}
p(z)^{\prime \prime}=-k h(z)-k h(z) p+\frac{\alpha}{3}\left(1-3 p+6 p^{2}-10 p^{3}+15 p^{4} \ldots\right) \\
+q\left(1-k^{2} \delta(z) \ldots\right) .
\end{array}
$$

To solve for the ripple $p(z)$ and the mean radius $A$ (which appears in the definitions of $\alpha$ and q), we decompose Eq. (23) into a pair of equations. Averaging Eq. (23),

$$
0=-k\langle h \rho\rangle+\frac{\alpha}{3}+2 \alpha\left\langle\rho^{2}\right\rangle-\frac{10}{3} \alpha\left\langle p^{3}\right\rangle+5 \alpha\left\langle\rho^{4}\right\rangle \ldots+q .
$$

Subtracting Eq. (24) from (23),

$$
\begin{array}{r}
\rho^{\prime \prime}=-\mathrm{kh}(\mathrm{z})-\mathrm{k}\{\mathrm{h} \rho\}-\alpha \rho+2 \alpha\left\{\mathrm{p}^{2}\right\}-\frac{10}{3} \alpha\left\{\mathrm{p}^{3}\right\}+5 \alpha\left\{\mathrm{\rho}^{4}\right\} \ldots \\
-\mathrm{qk} \delta(\mathrm{z}) \ldots,
\end{array}
$$

with $\{.$.$\} from Eq. (5). There are now two equations, each$ containing $A$ and $\rho(z)$. Because of our periodicity constraint these have the essence of the K-V equations (1) and (2).

\section{ITERATIVE SOLUTION OF K-V EQUATIONS}

On the right of Eq. (25), the $\mathrm{kh}(\mathrm{z})$ term dominates the terms involving the unknown function $\rho(z)$. They are omitted for the initial integrations, which give $P_{(0)}$. Then we insert $p_{(0)}$ into (25) and integrate again to get $P_{(1)}$. The process is repeated for $P_{(2)}$. The resulting terms of greatest significance are:

$$
\begin{gathered}
\rho_{(0)}=-k g, \\
\rho_{(1)}=\rho_{(0)}+\alpha k \iint g+k^{2} \delta+\frac{10}{3} \alpha k^{3} \iint g^{3}, \\
\rho_{(2)}=\rho_{(1)}-\alpha^{2} k \iiint \int g-k^{3} \iint h \delta-2 \alpha k^{3} \iint g \delta .
\end{gathered}
$$

To complete the approximate solution of the $\mathrm{K}-\mathrm{V}$ equations, $p(z)$ from $\mathrm{Eq}$. (26) is put in the matching equation (24). From 

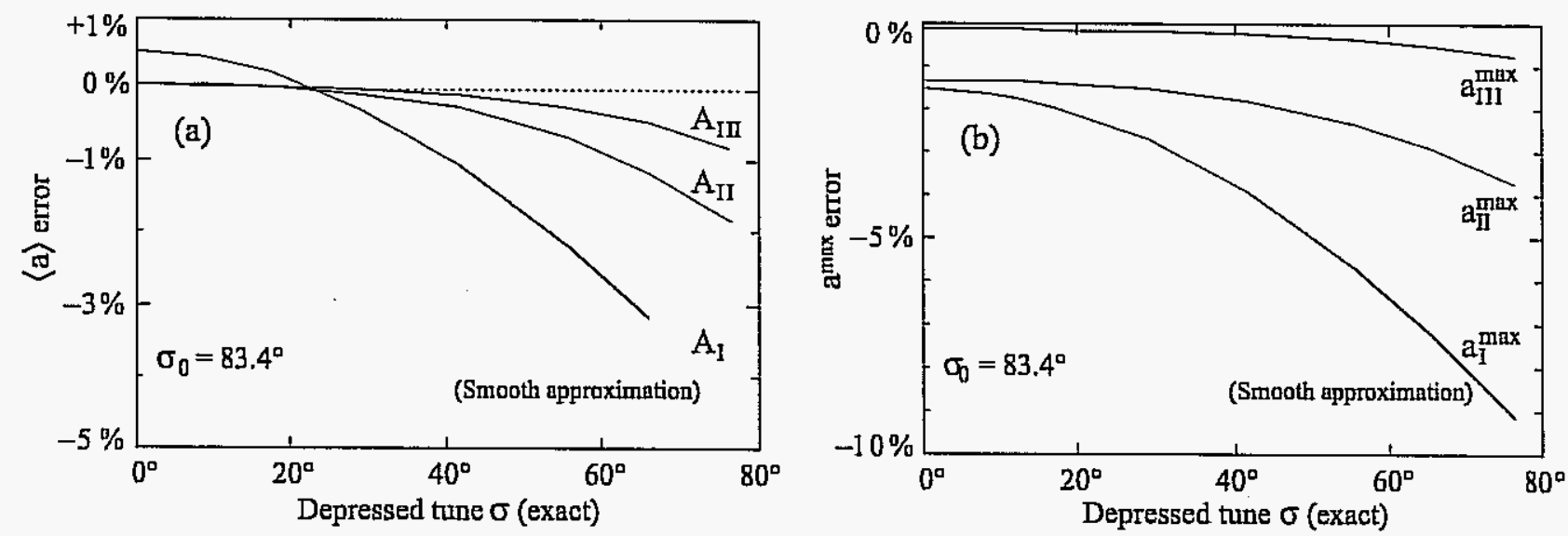

Fig. 1. Accuracy of (a) mean radius A from Eqs. (30), (32), or (33) and (b) maximum radius $a^{\max }$ from Eqs. (36), (37), or (38). Input quantities are $Q, \in$, and quadrupole voltage $V_{Q}$ (proportional to $K$ ). Quadrupole dimensions, etc., are in Ref. [8]. $V_{Q}$, fixed at $20 \mathrm{kV}$, gives phase advance $\sigma_{0}=83.37^{\circ} ; \in, Q$ are varied so that depressed tune $\sigma$ ranges between $0^{\circ}$ and $76.5^{\circ}$; exact $\sigma_{0}$ and $\sigma$ are obtained numerically.

Eq. (26) we discarded items, such as $2 \alpha \mathrm{k}^{2} \iint \mathrm{g}^{2}$, that would give terms in (24) higher than third power in the parameters $\mathrm{k}^{2}, \alpha$, and q. A miniscule term, $q \mathrm{k}^{2} \iint \delta(\mathrm{z})$, in $\rho_{(0)}$ is also omitted.

The order of a term in the matching equation is reckoned by counting the number of factors $k^{2}, \alpha$, and $q$. (These would become small parameters in a non-dimensional formalism [8]. Here, we prefer to retain physical units for length z, etc.)

Third Order: Inserting Eq. (26) into Eq. (24) yields seven terms [8] through third order. Some terms combine, with result

where

$$
\mathrm{K}_{t}^{\text {eff }}-\frac{E_{\text {III }}^{2}}{\mathrm{~A}_{\text {III }}^{4}}-\frac{\mathrm{Q}}{\mathrm{A}_{\text {III }}^{2}}=0 \text {, }
$$

$$
\begin{gathered}
\mathrm{K}_{\dagger}^{\text {eff }} \equiv\left\langle\left[\int \mathrm{K}(\mathrm{z})\right]^{2}\right\rangle\left[1+\frac{1}{24} \Phi\left(1+\frac{20}{27} \mathrm{c}_{3}\right)\right] \\
\epsilon_{\mathrm{II}}{ }^{2} \equiv \epsilon^{2}\left[1+\Phi\left(1+\frac{1}{2} \Phi+3 \beta_{\mathrm{I}}\right)\right] .
\end{gathered}
$$

Here $c_{3}$ is of order unity [8]. Roman-numeral subscripts on A and $E$ signify the order of approximation-third order in this case. The subscript on $\beta \sim A^{-4}$ indicates that $A,[E q$. (33)] is used to approximate $A$. The matching equation (27) is in the standard form of the smooth approximation, Eq. (33), and can be solved to find the third-order A:

$$
\mathrm{A}_{\mathrm{III}}{ }^{2}=\left(\mathrm{Q}^{2} / 2 \mathrm{~K}_{\dagger}^{\mathrm{eff}}\right)+\left[\left(\mathrm{Q} / 2 \mathrm{~K}_{\dagger}^{\mathrm{eff}}\right)^{2}+\epsilon_{\mathrm{II}}{ }^{2} / \mathrm{K}_{\dagger}^{\mathrm{eff}}\right]^{1 / 2} \text {. }
$$

If the input quantity is the mean radius $A_{\text {inp, }}$ then Eq. (27) gives the allowable $Q$ to third order,

$$
\mathrm{Q}_{\mathrm{III}}=\mathrm{A}_{\mathrm{inp}}{ }^{2} \mathrm{~K}_{+}^{\mathrm{eff}}-\epsilon_{\mathrm{mI}}^{2} / \mathrm{A}_{\text {inp }}{ }^{2} \text {. }
$$

Second Order: There are two second-order terms. One yields the correction to $\mathrm{K}^{\text {eff }}$ seen in $\mathrm{Eq}$. (28). The other term is $\alpha \mathrm{k}^{2}\left\langle\mathrm{~g}^{2}\right\rangle$, or, using definition (18), $\frac{\alpha}{3} \Phi$. We define

and get

$$
\epsilon_{\mathrm{II}}^{2} \equiv \epsilon^{2}(1+\Phi) \text {, }
$$

$$
\mathrm{K}_{\dagger}^{\mathrm{eff}}-\frac{E_{\mathrm{II}}^{2}}{A_{\mathrm{II}}^{4}}-\frac{\mathrm{Q}}{A_{\mathrm{II}}^{2}}=0 \text {. }
$$

Eq. (32) can be solved for $A_{n}$ or $Q_{n}$ in the same way as for the third order, giving useful approximations when $K(z)$ and $E$ produce $\sigma_{0}$ and $\sigma$ less than about $80^{\circ}$.

First Order. The three terms of lowest order produce what is called the first-order matching equation in this paper (Ref. [7] used another terminology). This is the classic smooth approximation. These terms give $\mathrm{k}^{2}\left\langle[\mathrm{~h}]^{2}\right\rangle=\alpha / 3+\mathrm{q}$, or, using the definitions (15), (16), and (17)

$$
\mathrm{K}^{\text {eff }}-\frac{\epsilon^{2}}{\mathrm{~A}_{\mathrm{I}}^{4}}-\frac{\mathrm{Q}}{\mathrm{A}_{\mathrm{I}}^{2}}=0 .
$$

First, second, and third-order results for A, from (33), (32) and (30), are plotted in Fig. 1a. The smooth approximation is relatively inaccurate except near the point where its error curve crosses the $0 \%$ line.

\section{MAXIMUM RADIUS}

Knowing the matched mean radius $A$, one can complete the solution for the envelope $a(z)=A[1+p(z)]$ using $p(z)$ from Eq. (26); $b(z)$ can be found by changing the sign of the terms that contain odd powers of $k$.

Some terms of Eq. (26) can be written in exact form [8] for models such as FODO, but Fourier expansion is more useful in general:

$$
h(z)=h_{1}\left[\cos \frac{\pi z}{L}+\frac{1}{3} c_{3} \cos 3 \frac{\pi z}{L}+\frac{1}{5} c_{5} \cos 5 \frac{\pi z}{L} \ldots\right] .
$$

Values (usually of order unity) of $h_{1}$ and $c_{n}$ for both FODO and smooth profiles are given in Ref. [8]. With the definition

we have

$$
\beta_{I} \equiv 3 \frac{L^{2}}{\pi^{2}} \frac{E^{2}}{A_{I}^{4}}
$$

$$
\begin{aligned}
a_{\text {II }}^{\max }=A_{\text {II }}\left[1+\rho_{m}\left(1+\frac{1}{27} c_{3}\right.\right. & \left.+\frac{1}{125} c_{5}\right)+\frac{1}{8} p_{m}^{2}\left(1+\frac{25}{54} c_{3}\right) \\
& \left.+\beta_{1} p_{m}\left(1+\frac{5}{2} p_{m}^{2}+\beta_{1}\right)\right]
\end{aligned}
$$

using results from Ref [8]. The accuracy of Eq. (36) is shown in Fig. 1b, along with that of the truncations 

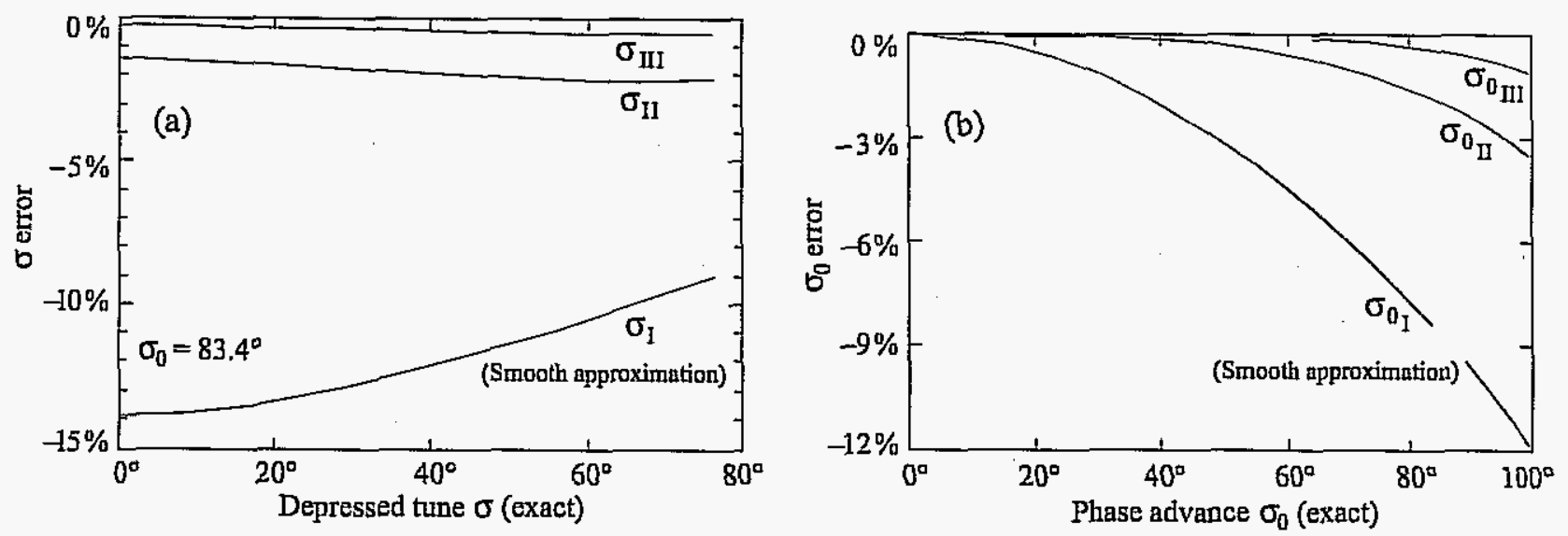

Fig. 2. (a) Accuracy of depressed tune $\sigma$ from Eqs. (40), (41), and (42). $V_{Q}$ is fixed at $20 \mathrm{kV}$ as in Fig. 1 .

(b) Accuracy of phase advance $\sigma_{0}$ from Eqs. (43), (44), and (45). $V_{Q}$ ranges from $5 \mathrm{kV}$ to about $22 \mathrm{kV}$.

$$
a_{I I}^{\max }=A_{I I}\left[1+p_{m}\left(1+\frac{1}{27} c_{3}+\frac{1}{125} c_{5}\right)+\beta_{I} p_{m}\right]
$$

and (the smooth approximation)

$$
\mathrm{a}_{\mathrm{I}}^{\max }=A_{\mathrm{I}}\left[1+p_{\mathrm{m}}\right] \text {. }
$$

\section{PHASE ADVANCES}

From the well-known phase-amplitude result [9], the phase advance per quadrupole cell of length $2 \mathrm{~L}$ is

$$
\sigma=E \int_{0}^{2 L} \frac{d z}{a^{2}}=2 L \in\left\langle a^{-2}\right\rangle \text {. }
$$

We approximate $a(z)$ by $A_{m}[1+p(z)]$ with $A_{m}$ from Eq. (30) and $p(z)$ to third order from Eq. (26). Subscripts are omitted for brevity. Expanding $\mathrm{a}^{-2}$ and taking the average gives

$$
\sigma=2 L \frac{E}{A_{\mathrm{II}}^{2}}\left[1+3\left\langle p^{2}\right\rangle-4\left\langle\rho^{3}\right\rangle+5\left\langle\rho^{4}\right\rangle-\cdots\right] .
$$

(The $2 p$ term has zero average by definition.) Ref. [8] shows that to third-order accuracy

$$
\sigma_{\mathrm{III}}=2 \mathrm{~L} \frac{\epsilon}{\mathrm{A}_{\mathrm{II}}^{2}}\left[1+\Phi\left(1+\frac{3}{4} \Phi+2 \beta_{1}\right)\right] .
$$

Enors with respect to exact values from simulations are shown in Fig. 2a. Useful accuracy is retained after dropping two terms and using $A_{I I}$ from Eq. (32):

$$
\sigma_{\mathrm{n}}=2 \mathrm{~L} \frac{\epsilon}{\mathrm{A}_{\mathrm{II}}^{2}}(1+\Phi) \text {. }
$$

Figure $2 a$ shows large errors for the first-order result (smooth approximation):

$$
\sigma_{\mathrm{I}}=2 \mathrm{~L} \frac{E}{\mathrm{~A}_{\mathrm{I}}^{2}} \text {. }
$$

The undepressed $\sigma_{0}$ is found by setting $Q=0$ in Eq. (27), then eliminating $\in$ from Eq. (40). Details are in Ref. [8]. The result is

$$
\sigma_{0 \mathrm{II}}=2 \mathrm{~L}\left(\mathrm{~K}_{\dagger}^{\mathrm{eff}}\right)^{1 / 2}\left[1+\frac{1}{2} \Phi+\frac{7}{8} \Phi^{2}\right]
$$

This equation is used to calculate $\sigma_{0}$ as a function of the strength of the quadrupole field gradient. Figure $2 b$ shows its accuracy and also illustrates the second-order case

$$
\sigma_{0_{\mathrm{n}}}=2 \mathrm{~L}\left(\mathrm{~K}_{\dagger}^{\mathrm{eff}}\right)^{1 / 2}\left[1+\frac{1}{2} \Phi\right]
$$

and the smooth approximation,

$$
\sigma_{0_{1}}=2 \mathrm{~L}\left(\mathrm{~K}^{\mathrm{eff}}\right)^{1 / 2}
$$

\section{ACKNOWLEDGEMENTS}

E.P. Lee and L.L. Lodestro offered helpful suggestions. Supported in part by the U.S. Department of Energy under Contract No. DE-AC03-76SF00098.

\section{REFERENCES}

[1] Kapchinskij and V.V. Vladimirskij, Proc. Int. Conf. on High Energy Accel, and Instrum. (CERN, 1959), p. 274.

[2] M. Reiser, Particle Accelerators 8, 167 (1978).

[3] J. Struckmeier and M. Reiser, Particle Accelerators 14, 227 (1984).

[4] R.C. Davidson, Physics of Nonneutral Plasmas, N.Y., 1990; R.C. Davidson and Q. Qian, Phys. Plasmas 1, 3104 (1994).

[5] E.P. Lee, T.J. Fessenden, and L.J. Laslett, IEEE Trans. Nuc. Sci. NS-32, 2489 (1985).

[0] E.P. Lee, Particle Accelerators 52 (1996).

[7] O.A. Anderson, Particle Accelerators 52, 133 (1996); O.A. Anderson, Lawrence Berkeley Laboratory report LBL-261233 (Revised), 1995.

[8] For derivation of equations, etc., see the appendices to: O.A. Anderson, LBNL report LBNL-57388, 2005 (to be submitted to Accel. and Beams).

[9] E.D. Courant and H.S. Snyder, Ann. of Phys. 3, 1 (1958). 